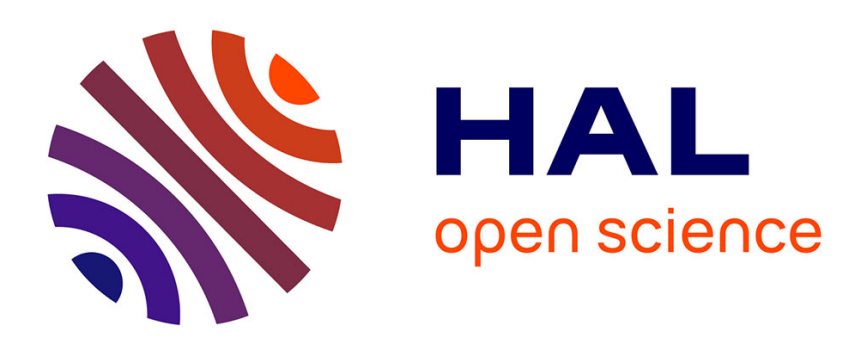

\title{
Pitfalls in electrodiagnosis of Guillain-Barré syndrome subtypes
}

Antonino Uncini, Claudia Manzoli, Francesca Notturno, Margherita Capasso

\section{To cite this version:}

Antonino Uncini, Claudia Manzoli, Francesca Notturno, Margherita Capasso. Pitfalls in electrodiagnosis of Guillain-Barré syndrome subtypes. Journal of Neurology, Neurosurgery and Psychiatry, 2010, 81 (10), pp.1157. 10.1136/jnnp.2010.208538 . hal-00579487

\section{HAL Id: hal-00579487 \\ https://hal.science/hal-00579487}

Submitted on 24 Mar 2011

HAL is a multi-disciplinary open access archive for the deposit and dissemination of scientific research documents, whether they are published or not. The documents may come from teaching and research institutions in France or abroad, or from public or private research centers.
L'archive ouverte pluridisciplinaire HAL, est destinée au dépôt et à la diffusion de documents scientifiques de niveau recherche, publiés ou non, émanant des établissements d'enseignement et de recherche français ou étrangers, des laboratoires publics ou privés. 


\title{
Pitfalls in electrodiagnosis of Guillain-Barré syndrome subtypes
}

\author{
Antonino Uncini ${ }^{1}$, Claudia Manzoli ${ }^{1}$, Francesca Notturno ${ }^{1,2}$, Margherita Capasso ${ }^{1}$ \\ ${ }^{1}$ Department of Human Motor Sciences, University "G. d'Annunzio", and \\ Neuromuscular Diseases Unit, Institute of Aging (Ce.S.I), Foundation University "G. \\ d'Annunzio", Chieti-Pescara, Italy \\ ${ }^{2}$ Institute of Advanced Biomedical Technologies (ITAB), Foundation University "G. \\ d'Annunzio",Chieti-Pescara, Italy
}

Word count: 3272

Key Words: Guillain-Barrè Syndrome; clinical neurophysiology

\section{Corresponding author:}

Prof. Antonino Uncini

Clinica Neurologica, Ospedale "SS. Annunziata",

Via dei Vestini, 66013, Chieti, Italy.

Tel: +390871 358584; Fax: +390871 562026

e-mail: uncini@unich.it 


\section{ABSTRACT}

Objective: To electrophysiologically classify an Italian Guillain Barré syndrome (GBS) population into demyelinating and axonal subtypes, to investigate how serial recordings changed the classification and to underline the pitfalls in electrodiagnosis of GBS subtypes.

Methods: We applied two current electrodiagnostic criteria sets for demyelinating and axonal GBS subtypes in 55 patients who had at least two serial recordings in three motor and sensory nerves.

Results: At first test electrodiagnosis was almost identical with both criteria: $65-67 \%$ of patients were classifiable as acute inflammatory demyelinating polyradiculoneuropathy (AIDP), $18 \%$ as axonal GBS, and $14-16 \%$ were equivocal. At follow-up $24 \%$ of patients changed classification: AIDP decreased to 58\%, axonal GBS increased to $38 \%$ and equivocal patients decreased to $4 \%$. The majority of shifts were from AIDP and equivocal groups to axonal GBS and the main reason was the recognition by serial recordings of the reversible conduction failure and of the lengthdependent compound muscle action potential amplitude reduction patterns as expression of axonal pathology.

Conclusions: Axonal GBS is pathophysiologically characterized not only by axonal degeneration but also by reversible conduction failure at the axolemma of Ranvier node. The lack of distinction among demyelinating conduction block, reversible conduction failure and length-dependent compound muscle action potential amplitude reduction may fallaciously classify patients with axonal GBS as AIDP. Serial electrophysiologic studies are mandatory for proper diagnosis of GBS subtypes and the identification of pathophysiological mechanisms of muscle weakness. More reliable electrodiagnostic criteria taking into consideration the reversible conduction failure pattern should be devised. 


\section{INTRODUCTION}

Guillain-Barré syndrome (GBS) is divided in three major subtypes: acute inflammatory demyelinating polyradiculoneuropathy (AIDP), acute motor axonal neuropathy (AMAN) and acute motor and sensory axonal neuropathy (AMSAN). AIDP is most frequent in Western countries whereas AMAN is common in China and Japan.[1-5] AMAN and AMSAN have been associated with antecedent Campylobacter jejuni (C. jejuni) infection and autoantibodies to gangliosides especially to GM1 and GD1a.[2-7] In AMAN and AMSAN the pathology is consistent with an antibody-mediated primary axonal damage at the Ranvier node.[8-

\section{1]}

AIDP, AMAN and AMSAN are difficult to distinguish on clinical grounds and electrophysiology plays a determinant role in GBS diagnosis, classification and in establishing the prognosis. AMAN is currently diagnosed by absence of demyelinating features and decrease of distal compound muscle action potential amplitude.[3,4] However some GBS patients with IgG to gangliosides showed besides axonal features rapid resolution of conduction block (CB) and conduction slowing without development of temporal dispersion or restoration of F-waves without increased latency.[12-15] These findings were thought to be incompatible with demyelination and remyelination and indicated that AMAN is characterized not only by axonal degeneration but also by "reversible conduction failure" possibly induced by anti-ganglioside antibodies at the axolemma of the Ranvier node.[12,15] The aim of this study was to electrophysiologically classify an Italian GBS population according to the current criteria, to investigate how serial recordings changed the initial classification and to underline the possible pitfalls in electrodiagnosis of GBS subtypes. 


\section{METHODS}

\section{Patients}

We reviewed the clinical and electrophysiological records of patients discharged with the diagnosis of GBS from the University Hospital of Chieti between January 1995 and June 2009 and selected patients with progressive weakness of more than one limb with or without sensory symptoms or signs, who had at least two electrophysiological recordings in at least three motor and three sensory nerves. Fifty-five patients fulfilled these requirements. All patients signed an informed consent for utilization of personal data, storage and assay of biological materials for research purposes.

\section{Electrophysiology}

Motor conduction studies were performed by a Nicolet Viking IV machine (Nicolet, USA). Amplitude and duration of negative peak of compound muscle action potential (CMAP) from distal (dCMAP) and proximal (pCMAP) stimulation, conduction velocity $(\mathrm{CV})$, distal motor latency (DML) and minimal F-wave latency were measured. Sensory studies were performed antidromically in median and ulnar nerves, orthodromically in sural nerve. Amplitudes of sensory nerve action potentials (SNAPs) were measured baseline to negative peak. For DML, CV and F wave latency we defined the upper and lower limit of normal as the mean $\pm 2.5 \mathrm{SD}$ of control values of our laboratory. For CMAP and SNAP amplitude the lower limit of normal was calculated as the mean \pm 2.5 SD of the logarithmically transformed amplitudes of the controls. Electrophysiological findings in patients were expressed as percentages of upper and lower limits of controls. In serial recordings of the same patients dCMAP amplitude was considered significantly increased when higher than $50 \%$ of the value found at the first study. Electrophysiology was performed in each patient at least 
twice in the same motor and sensory nerves. The total number of tests was 170 . The first test was performed within 15 days from onset of symptom (mean 9 days; range 2-15 days) and the results employed for the electrodiagnosis at first study. When a patient had more than one follow-up test the recording which we considered most informative was utilized for the final electrodiagnosis. The mean interval between disease onset and the most informative test was 28 days (range: 7-70 days). EMG of biceps brachialis, first dorsalis interosseous, quadriceps femoralis and tibialis anterior was performed in at least one follow up test and spontaneous activity was graded semi-quantitatively as absent, mild, moderate and abundant.

\section{Electrodiagnostic criteria}

In Table 1 are reported the electrodiagnostic criteria sets employed at initial test. In the Ho's criteria evidence of "unequivocal temporal dispersion" is enclosed among the parameters to assess demyelination.[3] As how much temporal dispersion of CMAP should be considered "unequivocal" was not defined we used an increased duration of negative peak of pCMAP $\geq 30 \%$ compared to dCMAP.[16] Hadden and colleagues replaced unequivocal temporal dispersion with $\mathrm{CB}$ defined as a pCMAP/dCMAP amplitude ratio less than 0.5.[4] AMSAN was diagnosed by absence of demyelinating features, as in the Ho's criteria, and reduction of sensory nerve action potential amplitude $<50 \%$ of lower limit of normal in at least two nerves. [8,17] Patient not fulfilling the criteria for AIDP, AMAN and AMSAN were classified as equivocal. The reasons for classification changes at follow-up are reported in the results section. 


\section{C. jejuni serology and definition of antecedent $C$. jejuni infection}

Sera from 51 of 55 patients were tested for the presence of $\operatorname{IgA}$ and $\operatorname{IgG}$ against $C$. jejuni by enzyme-linked immunosorbent assay (ELISA).[18] Subjects were considered to have had an antecedent $C$. jejuni infection if they had a positive stool culture for C. jejuni or a high optical densities for both IgA and IgG classes at dilution of 1:200 and 1:2000 and a definite history of a diarrheal illness within the previous 3 weeks before disease onset.

\section{Anti-ganglioside antibody testing}

Serum IgG and IgM to gangliosides GM1, GD1a, GD1b, were tested in acute phase sera by ELISA.[18] Serum was considered positive when showing antibody of IgM and $\mathrm{IgG}$ or only IgG class with titre $\geq 1: 400$.

\section{Statistical analysis}

Patients were grouped according to final electrodiagnosis, presence or not of antecedent $C$. jejuni infection, and presence or not of anti-ganglioside antibodies. Comparative analyses were made with Fisher's exact test. Results were considered significant if $\mathrm{p}<0.05$.

\section{RESULTS}

\section{Electrodiagnosis at first test}

At the first neurophysiologic test 37 of 55 patients (67\%) fulfilled the Hadden's criteria for AIDP, 10 patients (9 AMAN, 1 AMSAN) (18\%) for axonal GBS and 8 (14\%) were equivocal. According to Ho's criteria 36 patients (65\%) were classified as AIDP, 10 as axonal GBS (9 AMAN, 1 AMSAN) (18\%) and $9(16 \%)$ were equivocal (Fig. 1). 


\section{Follow-up and reasons for changes of electrodiagnosis}

Thirty patients initially classified as AIDP according to both criteria did not change classification at follow-up. Two patients, initially classified according to both criteria as equivocal, developed definite demyelinating features and were reclassified as AIDP. Fourteen of 32 patients (23\%) with final diagnosis of AIDP developed mild to moderate spontaneous activity in at least one muscle. The ten patients initially classified as axonal GBS showed at follow-up dCMAPs which substantially unchanged or further reduced, with some nerves becoming inexcitable, without the development of any demyelinating features and confirming a motor axonal degeneration pattern (Figs. 2A, 4A). All ten patients developed moderate to abundant spontaneous activity at EMG. Therefore none of these subjects changed the initial electrodiagnosis. Two patients according to Hadden's criteria and three patients according to Ho's criteria classified at the first study as equivocal developed amplitude reduction of dCMAPs and in one patient of SNAPs and were finally diagnosed as axonal subtypes. All three patients showed moderate to abundant spontaneous activity at EMG.

Three patients showed at the first study a progressive CMAP amplitude reduction along the nerve length (Fig. 2B) fulfilling in some segments the criterion for $\mathrm{CB}$ in at least one nerve. According to the Hadden's criteria, two patients were classified as AIDP whereas the other was classified as equivocal. According to Ho's criteria one patient was classified as AIDP and the other two were equivocal. Serial recordings showed that dCMAP amplitudes decreased becoming comparable to the pCMAPs with the disappearance of $\mathrm{CB}$ without development of excessive temporal dispersion 
or other features of demyelination (Fig. 2B). All three patients showed abundant spontaneous activity at EMG. We interpreted this length-dependent CMAP amplitude reduction as due to progressive loss of excitability in fibres undergoing axonal degeneration and these patients were finally classified as axonal GBS.[19-21] Partial motor CB was found in intermediate nerve segments in at least two nerves in four patients and in one nerve in other two patients. $\mathrm{CB}$ was localized in six ulnar nerves of four patients in the segment across the elbow with slow CV (range 24-38 $\mathrm{m} / \mathrm{s}$ ) (Figs. 2D, 3). In five of these six patients DMLs were prolonged in 11 nerves (mean $122 \%$ of ULN, range 110-147\%) reaching the cut-off for demyelination (Fig. 4B). Four of these six patients were classified on the basis of the first study according to both criteria as AIDP and two patients as equivocal. At serial recordings partial CB rapidly resolved without development of excessive temporal dispersion of dCMAPs or pCMAPs in all nerves (Figs. 2D, 3) and CV improved returning to the normal values in parallel with resolution of CB (Fig. 3). In 17 nerves dCMAP amplitude promptly increased with a mean of $141 \%$ (range $67-350 \%$ ) within a mean interval between the first study and follow-up of 9 days (range 6-17 days) (Figs. 2C, 4A). DMLs, when prolonged, returned to normal values within 3 weeks (Fig. 4B). The time course of electrophysiological abnormalities in the nerves of these six patients was clearly different from what found in nerves of patients with AIDP and AMAN with axonal degeneration (Figs. 2, 4). Only two of six patients developed mild spontaneous activity at EMG. These six patients were finally classified as axonal GBS with reversible conduction failure.[12-15]

\section{Final electrophysiological classification}

Because of the of better definition of electrophysiologic abnormalities at follow-up, the recognition of the reversible physiologic conduction failure and of the length- 
dependent CMAP reduction patterns as expressions of axonal pathology 13 subjects (24\%) changed classification (Fig. 1). Overall 32 patients (58\%) were finally classified as AIDP, $21(38 \%)$ as axonal GBS (18 AMAN, 3 AMSAN) and two (4\%) remained equivocal (Fig. 1). In the AIDP subtype $56 \%$ of patients had pain, $73 \%$ had paresthesias and $64 \%$ sensory loss. In AMAN subtype $38 \%$ of patients had pain, $16 \%$ had paresthesias and none sensory loss. All three AMSAN patients had paresthesias and sensory loss; pain was present in one patient.

\section{Relations between final electrodiagnosis, antecedent $C$. jejuni infection and anti- ganglioside antibodies}

Twenty-one of 51 (41\%) GBS patients had antecedent $C$. jeuni infection. Sixteen of 19 patients (84\%) with axonal GBS and 5 of 30 patients (17\%) with AIDP had antecedent $C$. jeuni infection. The difference was highly significant $(\mathrm{p}=0.000004)$. The two patients who remained equivocal at follow-up did not have antecedent $C$. jeuni infection.

Antibody to GM1 was present in eight AIDP and 12 axonal GBS (2 with reversible conduction failure). Antibody to GD1a was present in five AIDP and 13 axonal GBS (three with reversible conduction failure). Antibody to GD1b was present in six AIDP and eight axonal GBS (three with reversible conduction failure). Overall at least one antibody to gangliosides GM1, GD1a or GD1b was present in 28 of 55 (51\%) GBS patients, in 19 of $21(90 \%)$ axonal GBS and in 9 of 32 (28\%) AIDP patients. The difference was highly significant $(\mathrm{p}=0.000007)$. Eight of 10 patients who were classified as axonal GBS at first test and did not change classification at follow-up had antibodies to gangliosides. The three patients with the length-dependent CMAP amplidute reduction pattern and the six patients with the reversible conduction failure 
pattern had antibodies to gangliosides. The two patients who remained equivocal at follow-up did not have anti-ganglioside antibodies.

Fifteen of 19 (79\%) patients with axonal GBS had antecedent $C$. jejuni infection and at least one anti-ganglioside antibody. On the other hand only 4 (13\%) of 30 AIDP patients had antecedent $C$. jeuni infection and at least one of anti-ganglioside antibodies. The difference was highly significant (0.000002)

\section{DISCUSSION}

The percentages of patients with demyelinating and axonal GBS vary substantially in different series.[3,4,22,23] This may be due to genetic susceptibility, different triggering factors, electrophysiological criteria used and whether the electrodiagnosis was based on a single or serial studies. Ho and colleagues in 129 Chinese patients, classified by a single test, found 65\% AMAN, 24\% AIDP and 11\% unclassifiable patients.[3] Hadden and colleagues applied their criteria to 369 GBS patients from 11 Western countries examining the results of two electrophysiologic tests performed approximately four weeks apart.[4] At first test, $69 \%$ of patients met the criteria for AIDP and 3\% the criteria for AMAN; the remaining patients being equivocal or showing inexcitable nerves. At second test, although the final proportion of AIDP and AMAN were similar, many subjects changed classification. More recently in 51 Indian patients applying the Ho'criteria to the results of a single test $86 \%$ of patients had AIDP and 14\% axonal GBS whereas in 41 patients from Israel, using the results of two studies performed within 4 weeks from onset, the percentage of AIDP was 63 $\%$ and of axonal GBS 37\%.[22,23] In the GBS series we report electrodiagnosis was almost identical at the first test with both criteria but at follow-up 24\% patients 
changed classification. The main shifts being from AIDP and equivocal groups to the axonal group which resulted more than doubled. The principal reason was the individuation of CBs rapidly resolving without the development of dispersed CMAPs due to remyelinating, slow conducting, desynchronized components which are characteristic of the evolution of CB due to segmental demyelination.[24-26] This feature, denominated reversible conduction failure, has been described in some Japanese AMAN patients and in a GBS subtype named acute motor conduction block neuropathy $(\mathrm{AMCBN})$ and ascribed to an antibody mediated impaired physiological conduction at the axolemma of nodes of Ranvier.[12-15, 27-30] The pathophysiology of AMAN with axonal degeneration, AMAN with reversible conduction failure and AMCBN can be explained by the experimental model of axonal GBS. In the rabbit the immunopathologic cascade starts with IgG deposit at the axolemma of Ranvier nodes, is followed by complement activation with the final formation of the membrane attack complex, disruption of nodal sodium channel clusters, detachment of paranodal myelin terminal loops and lengthening of the nodal region.[31] All these changes lower the safety factor for impulse transmission and when transposed to humans suggest that at an early stage the immunologic reaction at nodal axolemma may induce a potentially reversible conduction failure. If the immune reaction progresses with the formation of membrane attack complex pores and calcium entry in the axon a process of cytoskeletal degradation and mitochondrial injury develops with axonal damage and Wallerian-like degeneration. To support a continuum between reversible conduction failure and axonal degeneration a reversibly reduced safety factor for impulse conduction has been documented by threshold tracking technique and refractory period studies in nerves of AMAN patients and one patient with AMCBN who progressed to axonal degeneration was reported.[32-34] The above considerations explain why AMAN patients do not necessarily have poor 
prognosis and may improve faster or slower according to the shares of axonal degeneration and reversible conduction failure, being the patients with AMAN with reversible conduction failure or AMCBN the ones with the best prognosis and complete recovery.[30,35,36]

An objection to the not demyelinating nature of reversible conduction failure might come from the observation that $\mathrm{CV}$ was slow at the site of $\mathrm{CB}$ in some nerves of patients we finally diagnosed as axonal GBS with reversible conduction failure. In these patients $\mathrm{CV}$ slowing was present from the first recording when $\mathrm{CB}$ was at maximum, $\mathrm{CV}$ rapidly increased with the decrease of $\mathrm{CB}$ and returned to normal range when $\mathrm{CB}$ had disappeared. Conduction slowing at $\mathrm{CB}$ sites may be a factitious phenomenon due to preferential block of large diameter fastest conducting fibres. The same explanation can be applied to the nerves showing prolonged DMLs which in sequential recordings quickly normalized in parallel with increased dCAMP amplitude. Alternatively sodium channel dysfunction, which is hypothesized to be at the basis of reversible conduction failure, may be responsible for conduction slowing. In human poisoning by tetrodotoxin slow CVs, increased DMLs and reduced dCMAP amplitudes recover in few days without temporal dispersion possibly because of an uniform involvement of all fibers in the nerve resulting in synchronous slowing of impulse conduction.[37]

Two patients were initially classified as AIDP because of progressive CMAP amplitude reduction along the nerve length fulfilling in some segments the criterion for CB. At follow up the dCMAP amplitude decreased and equalized the proximal CMAP without the development of temporal dispersion. This pattern was named by van der Meché and colleagues "length-dependent CMAP amplitude reduction" and initially thought to be due to demyelinating lesions scattered along the nerve followed by axonal degeneration.[38] However this pattern is also consistent with an immune- 
mediated primary axonal degeneration with progressive loss of excitability in fibres undergoing axonal degeneration.[19,20,21, 39] The dCMAP is initially greater than the pCMAP because the injured axons are inexcitable above the lesion site whereas they remain excitable distally. The inexcitability progresses over few days distally till it reaches the nerve terminals. Other possible explanations are that $\mathrm{CB}$ may be due to an axonal dysfunction, as in reversible conduction failure, which progresses to axonal degeneration or imputable to an adjunctive distal axonal involvement. In any case the damage should be considered axonal in nature.

Because of the recognition of reversible conduction failure and length dependent CMAP amplitude reduction patterns a total of 7 patients shifted from the AIDP and equivocal groups to axonal GBS and none from axonal GBS to AIDP. In the study by Hadden and colleagues six of ten patients initially classified in the axonal group were later reclassified as demyelinating, three equivocal and only one remained in the axonal group. Of the original demyelinating group 4\% become axonal and 5\% inexcitable.[4] This study was published in the same year of the paper on reversible conduction failure in axonal GBS and we think that, at least in part, the differences in results may be due to the lack of recognition of reversible conduction failure pattern. $[4,12]$

In the series we report antecedent C. jejuni infection and anti-ganglioside antibodies are more frequent in axonal GBS than in AIDP. Overall 79\% of axonal GBS but only $13 \%$ of AIDP had antecedent $C$. jejuni infection and anti-gangliosides antibodies. These results confirm that the three conditions: axonal GBS (including reversible conduction failure), antecedent $C$. jeuni infection and IgG to gangliosides GM1, GD1a, or GD1b are closely associated.[5] Hiraga and colleagues advanced the hypothesis that anti-ganglioside antibodies are associated to axonal degeneration or reversible conduction failure but not to true AIDP.[15] In our series all patients who 
shifted to the axonal group had anti-ganglioside antibodies. However $28 \%$ of patients finally classified as AIDP have anti-ganglioside antibodies. Reversible conduction failure is thought to be due to a transient attack of antibodies and complement limited at the excitable axolemma of Ranvier node but it is conceivable that an immunologic process, although specific, cannot have the "surgical" precision to completely spare the paranodal region. As a matter of fact detachment of terminal loops of paranodal myelin and lengthening of the nodal region has been shown in AMAN patients and in the experimental model.[10,11,31] This feature, although the primary pathology is on the axonal side, mimic paranodal demyelination and may explain the "grey zone" of patients with anti-ganglioside antibodies and some de-remyelinating features at electrophysiology fulfilling the criteria for AIDP.

This study demonstrates that in the early phase of GBS the distinction between AIDP and axonal GBS may be difficult or even impossible in some patients. Theoretically the Ho's criteria including excessive temporal dispersion but not CB should avoid the pitfalls due to reversible conduction failure and length-dependent CMAP amplitude reduction resulting more specific than Hadden's criteria for AIDP. However the fact that reversible conduction failure can also transiently slow CV and increase DML eliminates the advantage making the two criteria substantially equivalent. In conclusion in GBS only serial electrophysiological studies allow the identification of pathophysiological mechanisms of muscle weakness and the correct classification in subtypes. More reliable electrophysiological criteria taking into consideration the reversible conduction failure pattern should be devised and validated in large populations. 
TABLE 1. CRITERIA FOR ELECTROPHYSIOLOGICAL CLASSIFICATION AIDP

a) Ho et al.1985[3]

Patients must have one of the following in two or more nerves during the first 2 weeks of illness:

Motor conduction velocity $<90 \%$ LLN ( $<85 \%$ if dCMAP $<50 \%$ LLN)

Distal motor latency $>110 \%$ ULN $(>120 \%$ if dCMAP $<100 \%$ LLN)

Evidence of unequivocal temporal dispersion

F-response latency $>120 \%$

b) Hadden et al.1998[4]

At least one of the following in each of at least two nerves, or at least two of the following in one nerve if all others inexcitable and dCMAP $\geq 10 \%$ LLN:

Motor conduction velocity $<90 \%$ LLN $(<85 \%$ if dCMAP $<50 \%$ LLN)

Distal motor latency $>110 \%$ ULN $(>120 \%$ if dCMAP<100\% LLN)

pCMAP/dCMAP ratio $<0.5$ and dCMAP $\geq 20 \%$ LLN

F-response latency $>120 \%$

\section{AMAN}

c) Ho et al. 1995[3]

No evidence of demyelination as defined in $a$ dCMAP $<80 \%$ LLN

d) Hadden et al. 1998[4]

None of the features of demyelination in any nerve as defined in $b$ (except one demyelinating feature allowed in one nerve if dCMAP $<10 \%$ LLN) dCMAP $<80 \%$ LLN in at least 2 nerves 


\begin{abstract}
AMSAN
e) Feasby et al. 1993[8], Rees et al. 1995[17]

No evidence of demyelination as defined in $a$

dCMAP $<80 \%$ LLN in at least 2 nerves

SNAP $<50 \%$ LLN in at least 2 nerves

LLN: lower limit of normal; ULN: upper limit of normal; dCMAP: amplitude of compound muscle action potential after distal stimulation; pCMAP amplitude of compound muscle action potential after proximal stimulation; SNAP sensory nerve action potential.
\end{abstract}




\section{COMPETING INTERESTS}

This study was not sponsored by any company. Prof. Uncini received research support from Kedrion and payement for lectures by Pfizer.

\section{FUNDING}

This study was supported by a grant from Ministero della Salute (Ricerca Corrente 2006).

\section{COPYRIGHT LICENCE STATEMENT}

The Corresponding Author has the right to grant on behalf of all authors and does grant on behalf of all authors, an exclusive licence on a worldwide basis to the BMJ Publishing Group Ltd, and its Licensees to permit this article (if accepted) to be published in Journal of Neurology Neurosurgery and Psychiatry and any other BMJPGL products and to exploit all subsidiary rights, as set out in our licence. 


\section{REFERENCES}

1. McKhann GR, Cornblath DR, Griffin JW, et al. Acute motor axonal neuropathy: a frequent cause of acute flaccid paralysis in China. Ann Neurol $1993 ; 33: 333-42$.

2. Rees JH, Gregson NA, Hughes RAC. Anti-ganglioside GM1 antibody in Guillain-Barré syndrome and their relationship to Campylobacter jejuni infection. Ann Neurol 1995;38:809-16.

3. Ho TW, Mishu B, Li CY, et al. Guillain-Barré syndrome in Northen China. Relationship to Campylobacter jejuni infection and anti-glycolipid antibodies. Brain 1995;118:597-605.

4. Hadden RDM, Cornblath DR, Hughes RAC, et al. Electrophysiological classification of Guillain-Barré syndrome: clinical associations and outcome. Ann Neurol 1998;44:780-8.

5. Ogawara K, Kuwabara S, Mori M, et al. Axonal Guillain-Barré syndrome: relation to anti-ganglioside antibodies and Campylobacter iejuni infection in Japan. Ann Neurol 2000;48:624-31.

6. Ho TW, Willison HJ, Nachamkim I, et al. Anti-GD1a antibody is associated with axonal but not demyelinating forms of Guillain-Barré syndrome. Ann Neurol 1999;45:168-73.

7. Yuki N, Kuwabra S, Koga M, et al. Acute motor axonal neuropathy and acute motor-sensory neuropathy share a common immunological profile. J Neurol Sci 1999;168:121-26.

8. Feasby TE, Hahn AF, Brown WF, et al. Severe degeneration in acute Guillain-Barré syndrome evidence of two different mechanisms. J Neurol Sci 1993;116:185-92. 
9. Griffin JW, Li CY, Ho TW, et al. Guillain-Barré syndrome in Northen China: the spectrum of neuropathological changes in clinically defined cases. Brain 1995;118:577-95.

10. Griffin JW, Li CY, Ho TW et al. Early nodal changes in the acute motor axonal neuropathy pattern of Guillan-Barré sindrome. J Neurocytol 1996;25:3351.

11. Hafer-Macko C, Hsieh S-T, Li CY, et al. Acute motor axonal neuropathy: an antibody-mediated attack on axolemma. Ann Neurol 1996;40:635-44.

12. Kuwabara S, Yuki N, Koga M, et al. IgG anti-GM1 antibody is associated with reversible conduction failure and axonal degeneration in Guillain-Barré syndrome. Ann Neurol 1998;44:202-8.

13. Kuwabara S, Mori M, Ogawara K, et al. Axonal involvement at the common entrapment sites in Guillain-Barré syndrome with IgG anti-GM1 antibody. Muscle Nerve 1999;22:840-5.

14. Kuwabara S, Ogawara K, Mizobuchi K, et al. Isolated absence of F waves and proximal axonal dysfunction in Guillain-Barré syndrome with antiganglioside antibodies. J Neurol Neurosurg Psychiatry 2000;68:191-5.

15. Hiraga A, Kuwabara S, Ogawara K, et al. Patterns and serial changes in electrodiagnostic abnormalities of axonal Guillain-Barré syndrome. Neurology 2005;64:856-60.

16. Albers JW, Donofrio PD, McGonagle TK. Sequential electrodiagnostic abnormalities in acute inflammatory demyelinating polyneuropathy. Muscle Nerve 1985;8:528-39.

17. Rees JH, Soudain SE, Gregson NA, et al. Campylobacter Jejuni infection and Guillain-Barré syndrome. N Engl J Med 1995;33:1374-79. 
18. Caporale CM, Papola F, Fioroni MA, et al. Susceptibility to Guillain-Barrè is associated to polymorphisms of CD1 genes. J Neuroimmunol 2006;177:112-8.

19. Brown WF, Feasby TE, Hahn AF. The electrophysiological changes in the acute "axonal” form of Guillain-Barré syndrome. Muscle Nerve 1993;16:200-5.

20. Lugaresi A, Ragno M, Torrieri F, et al. Acute motor axonal neuropathy with high titer IgG and IgA anti-GD1a antibodies following Campylobacter enteritis. $J$ Neurol Sci 1997;147:193-200.

21. Uncini A, Capasso M. Acute motor conduction block neuropathy followed by axonal degeneration and poor recovery. Neurology 2006;67:543.

22. Kalita J, Misra UK, Das M. Neurophysiological criteria in the diagnosis of different clinical types of Guillain-Barré syndrome. J Neurol Neurosurg Psychiatry 2007;79:289-93.

23. Kushnir M, Klein C, Pollak L, et al. Evolving pattern of Guillain-Barré syndrome in a community hospital in Israel. Acta Neurol Scand 2008;117:347-50.

24. Saida K, Sumner AJ, Saida T, et al. Antiserum-mediated demyelination: relationship between remyelination and functional recovery. Ann Neurol $1980 ; 8: 12-24$.

25. Uncini A, England JD, Rhee EK, et al. Tellurium-induced demyelination: an electrophysiological and morphological study. Muscle Nerve 1988;11:871-79.

26. Clouston PDE, Kiers L, Zuniga G, et al. Quantitative analysis of the compound muscle action potential in early acute inflammatory demyelinating polyneuropathy. Electroencephalogr Clin Neurophysiol 1994;93:245-54.

27. White JR, Sachs GM, Gilchrist JM. Multifocal motor neuropathy with conduction block and Campylobacter jejuni. Neurology 1996;46:562-3. 
28. Sugie K, Murata K, Ikoma K, et al. A case of acute multifocal motor neuropathy with conduction block after Campylobacter jejuni enteritis. Rinsho Shinkeigaku 1998;38:42-5.

29. Susuki K, Johkura K, Yuki N, et al. Rapid resolution of nerve conduction blocks after plasmapheresis in Guillain-Barré syndrome associated with antiGM1b IgG antibody. J Neurol 2001;248:148-50.

30. Capasso M, Caporale CM, Pomilio F, et al. Acute motor conduction block neuropathy. Another Guillain-Barré syndrome variant. Neurology 2003;61:617-

22.

31. Susuki K, Rasband M, Tohyama K, et al. Anti-GM1 antibodies cause complement-mediated disruption of sodium channel clusters in peripheral motor nerve fibers. J Neurosci 2007;27:3956-67.

32. Kuwabara S, Ogawara K, Sung JK et al. Differences in membrane properties of axonal and demyelinating Guillain-Barré syndromes. Ann Neurol 2002;52:180-7.

33. Kuwabara S, Bostock H, Ogawara K et al. The refractory period of transmission is impaired in axonal Guillain-Barré syndrome. Muscle Nerve 2003;28:683-9.

34. Rajabally YA, Strens LHA, Abbott RJ. Acute motor conduction block neuropathy followed by axonal degeneration and poor recovery. Neurology 2006;66:287-8.

35. Kuwabara S, Mori M, Ogawara K, et al. Indicators of rapid recovery in Guillain Barré syndrome. J Neurol Neurosurg Psychiatry 2001;70:560-2.

36. Hiraga A, Mori M, Ogawara K, et al. Recovery patterns and long term prognosis for axonal Guillain-Barré syndrome. J Neurol Neurosurg Psychiatry 2005;76:719-722. 
37. Oda K, Araki K, Totoki T, Shibasaki H. Nerve conduction study of human tetrodoxication. Neurology 1989;39:743-45.

38. van der Meché FGA, Meulstee J, Vermeulen M, et al. Patterns of conduction failure in the Guillain-Barré syndrome. Brain 1988;111:405-16.

39. van der Meché FGA, Meulstee J, Kleyweg RP. Axonal damage in GuillainBarré syndrome. Muscle Nerve 1991;14:997-1002. 


\section{FIGURE LEGENDS}

\section{Figure 1. Electrodiagnosis of GBS patients at first study and after serial}

recordings.

The arrows indicate the directions of changes from a subtype to another and the numbers the subjects changing group.

\section{Figure 2. Patterns of motor conduction abnormalities.}

Superimposed compound muscle action potentials (CMAPs) recorded from the abductor digiti minimi after ulnar nerve stimulation at wrist, below-elbow and aboveelbow and from the abductor pollicis brevis after median nerve stimulation at wrist and elbow.

A) Acute motor axonal degeneration pattern. Ulnar nerve. Distal CMAP amplitude was already decreased $(4 \mathrm{mV})$ on day four and further decreased $(2 \mathrm{mV})$ on day 11. The patient had IgG anti-GM1 and anti-GD1a.

B) Length-dependent CMAP amplitude reduction pattern. Ulnar nerve. On day two there was a mild reduction (28\%) of CMAP amplitude from below elbow stimulation compared to wrist stimulation and an abnormal amplitude reduction of CMAP (65\%) from above elbow compared to below elbow stimulation. At day 11 all CMAP amplitudes were reduced and there was an abnormal amplitude reduction (64\%) of CMAP from below elbow stimulation compared to wrist whereas the CMAP amplitude drop across the was decreased (29\%). At day 26 distal CMAP amplitude 
was further decreased but amplitude reduction of CMAPs from proximal stimulation sites were no longer evident. The patient had IgG anti-GM1 and anti-GD1a.

C) Reversible distal conduction failure pattern. Median nerve. On day six distal and proximal CMAP amplitudes were reduced $(2.6 \mathrm{mV})$. On day 12 distal CMAP was $142 \%$ increased returning within the normal range. There was no excessive temporal dispersion of proximal or distal CMAP in all recordings from day six to day 25 . The patient had IgG anti-GD1b.

D) Reversible conduction failure pattern in intermediate nerve segments. Ulnar nerve. On day ten there was a partial CB across the elbow which improved on day 20 and resolved at day 27 without the development of excessive temporal dispersion. The patient had IgG anti-GM1, anti-GD1a and anti-GD1b.

E) Acute inflammatory demyelinating neuropathy pattern. Ulnar nerve. On day two all conduction parameters were normal. On day 14 all CMAPs were dispersed, distal CMAP amplitude was greatly reduced $(1 \mathrm{mV})$, distal motor latency was increased (5.7 $\mathrm{ms}$ ), the CMAP amplitude ratio between below-elbow and wrist stimulation was 0.2 and conduction velocities were reduced $(20 \mathrm{~m} / \mathrm{s}$ in the below-elbow wrist segment and $26 \mathrm{~m} / \mathrm{s}$ across the elbow). On day 40 the CMAP amplitude ratio between below-elbow and wrist stimulation was 0.5 but all CMAPs were still reduced in amplitude and dispersed, DML was further increased (7.2 ms) and conduction velocities reduced (19 $\mathrm{m} / \mathrm{s}$ in the below-elbow wrist segment and $16 \mathrm{~m} / \mathrm{s}$ across the elbow). The patient did not have anti-ganglioside antibodies. 
Figure 3. Serial electrophysiologic findings in ulnar nerves of two patients with AMAN and reversible conduction failure

In ordinates are: A) ratios between amplitudes of proximal (above elbow) and distal (below elbow) compound muscle action potential (P/D CMAP); B) P/D CMAP duration ratios; $\mathrm{C})$ conduction velocity $(\mathrm{CV})$ in the segment above-below elbow (continuous line) and below-elbow wrist (dashed lines). In abscissae are days. Time 0 is the time of the first recordings which were done between day 2 and 7 after the disease onset. CVs improved in above-below elbow segment in parallel with the resolution of conduction block without development of excessive temporal dispersion of proximal CMAPs. These patients had anti-ganglioside antibodies.

Figure 4. Serial electrophysiologic findings in patients with axonal GBS and AIDP

In abscissae are three time intervals of recordings after disease onset: T1=1-10 days, $\mathrm{T} 2=11-20$ days, $\mathrm{T} 3=21-30$ days. In ordinates: A) distal compound muscle action potential (dCMAP) amplitudes expressed as percentages of values at first recordings considered $100 \%$. Linerar graphs of 18 nerves of patients with AIDP without antiganglioside antibodies, 17 nerves of patients with AMAN and reversible distal conduction failure (AMAN RCF) and anti-ganglioside antibodies and 10 nerves of patients with AMAN and axonal degeneration (AMAN AX DEG). In ordinates: B) distal motor latencies (DMLs) expressed as percentages of upper limits of controls. Linear graphs 18 nerves of patients with AIDP without anti-ganglioside antibodies, 11 nerves of patients with AMAN and reversible distal conduction failure (AMAN RCF) prolonged DMLs and anti-ganglioside antibodies and 10 nerves of patients with AMAN and axonal degeneration (AMAN AX DEG) and anti-ganglioside antibodies. Vertical bars are standard errors. 
C) CMAPs recorded from the extensor digitorum brevis muscle after stimulation of peroneal nerve at ankle, below-fibular head and above-fibular head in a patient with antibodies to GD1b. On day six DML is prolonged and dCMAP amplitude reduced (1.6 mV). On day $12 \mathrm{DML}$ is slightly prolonged and dCMAP amplitude is $106 \%$ increased. On day $25 \mathrm{DML}$ is in the normal range and dCMAP amplitude is $150 \%$ increased compared to day six. 


\section{$\mathbf{A}$}

day 4 B

day $2 \mathbf{C}$

day $10 \mathbf{E}$

day 2

in

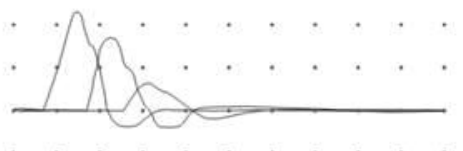

day 11

day 11
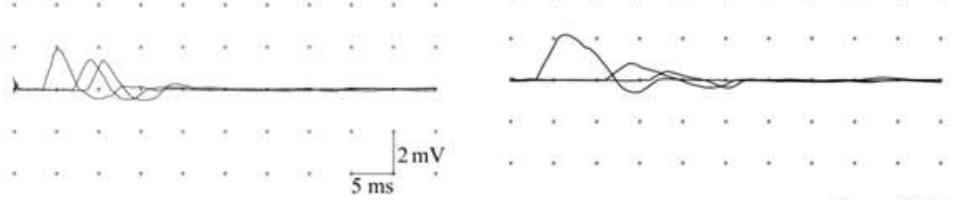

day 26

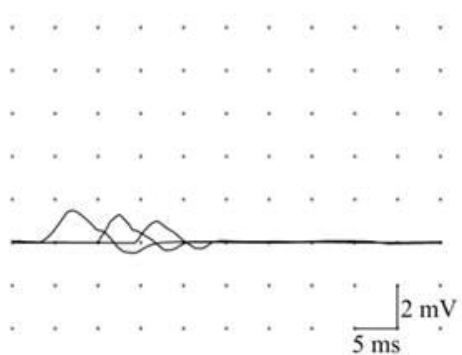

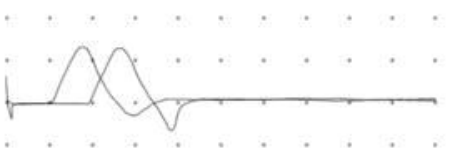

day 12

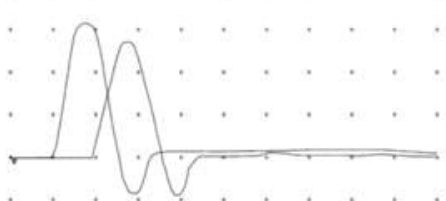

day 25

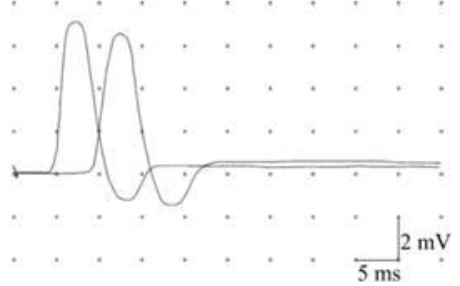

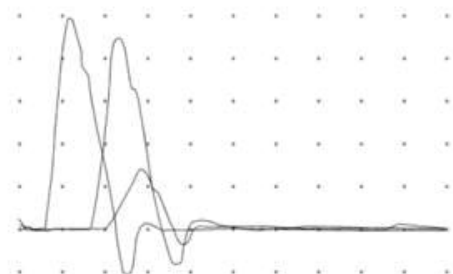

day 20

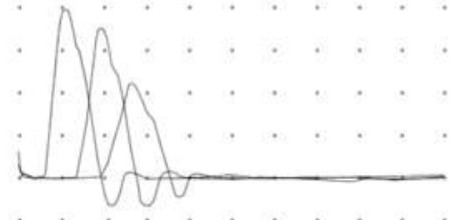

day 27

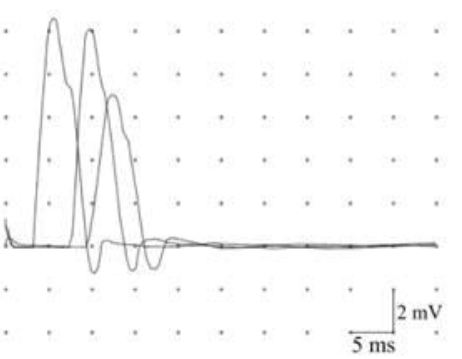

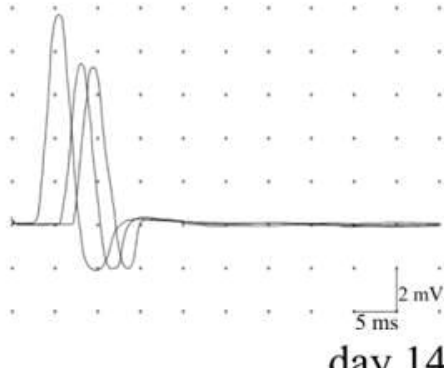

day 14
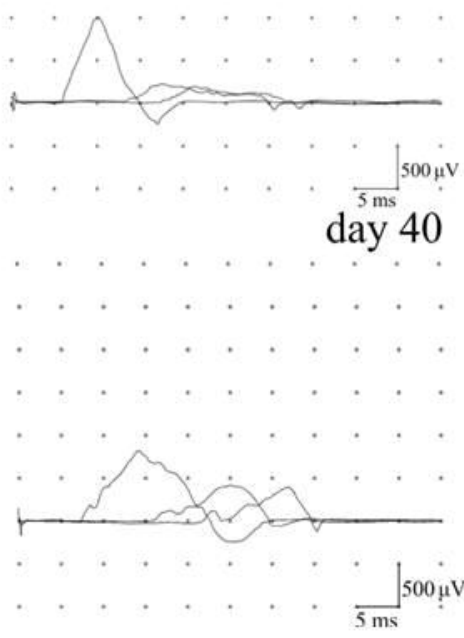
A

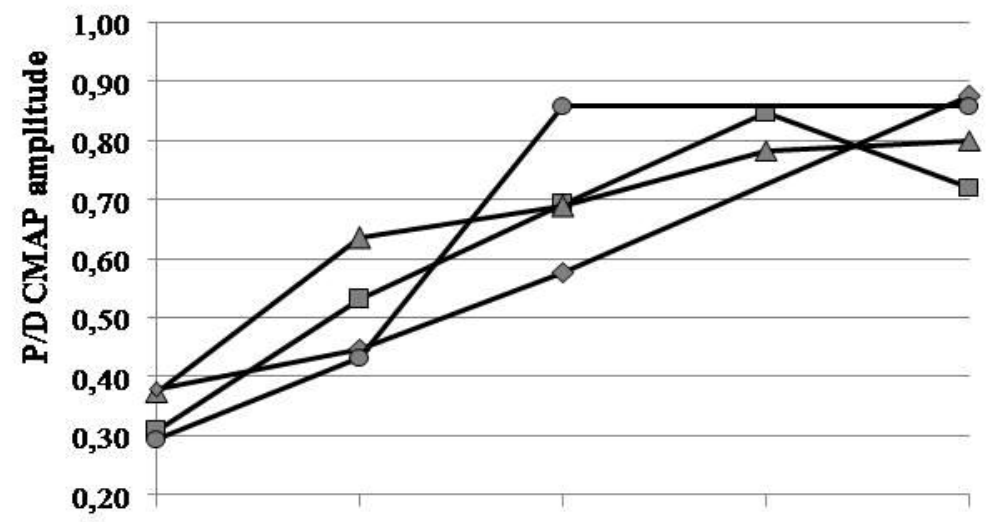

B

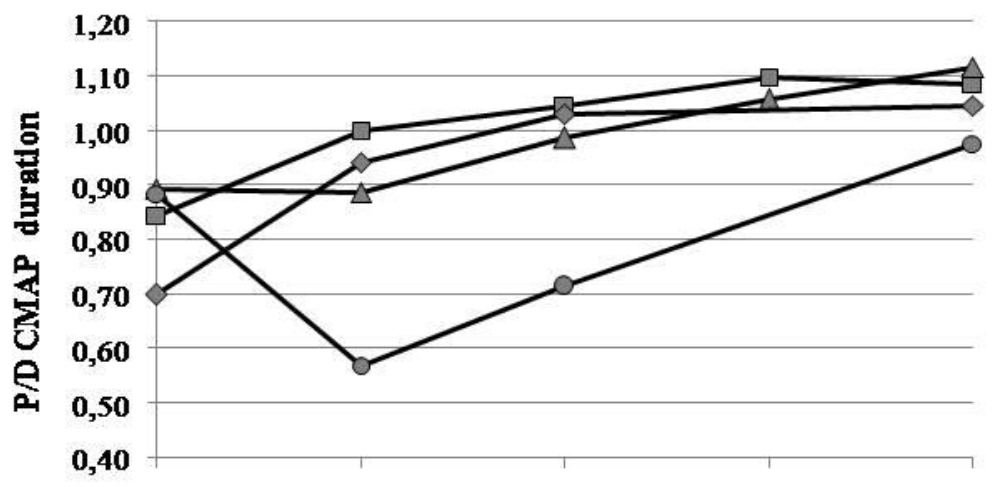

C

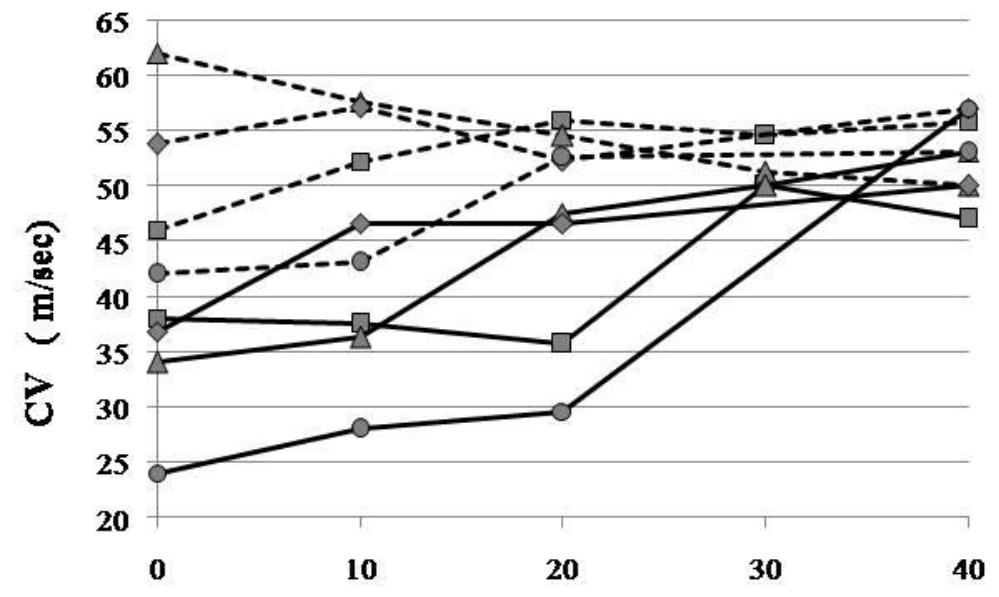




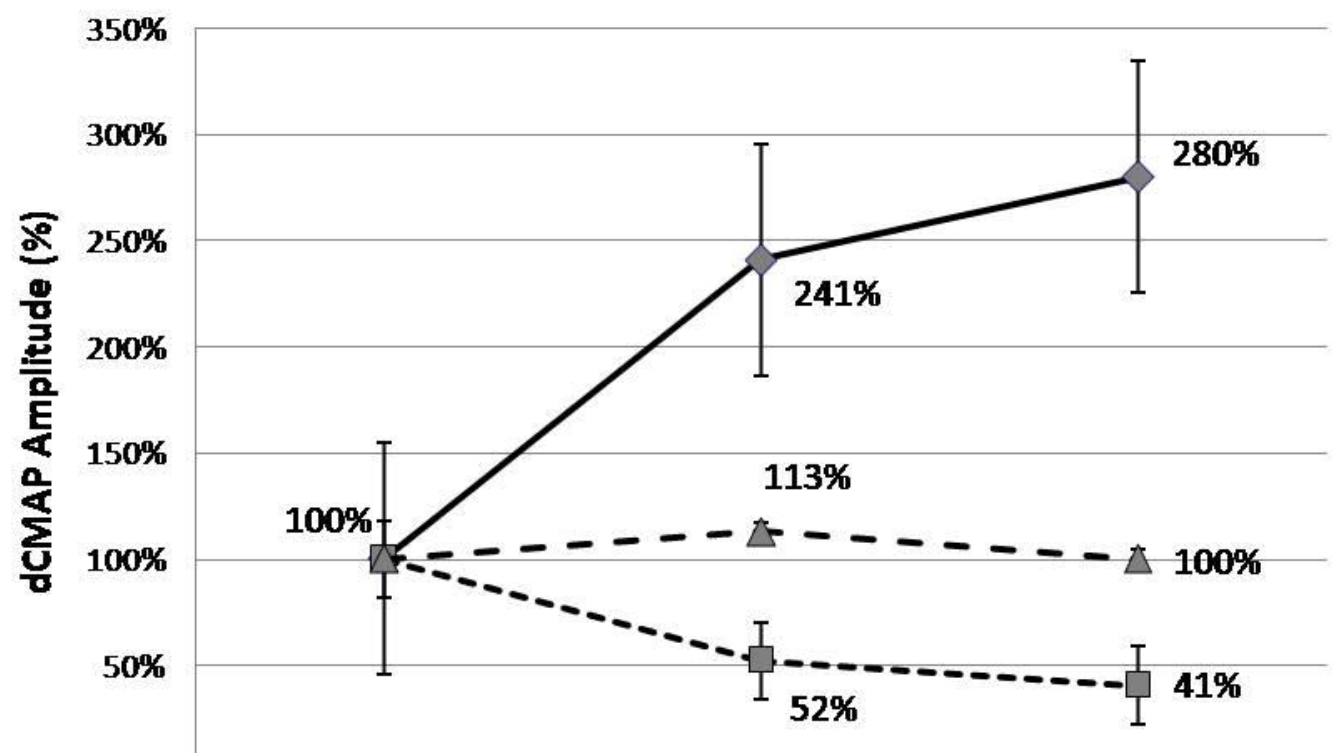

$0 \%$

I1

I2

T3

B

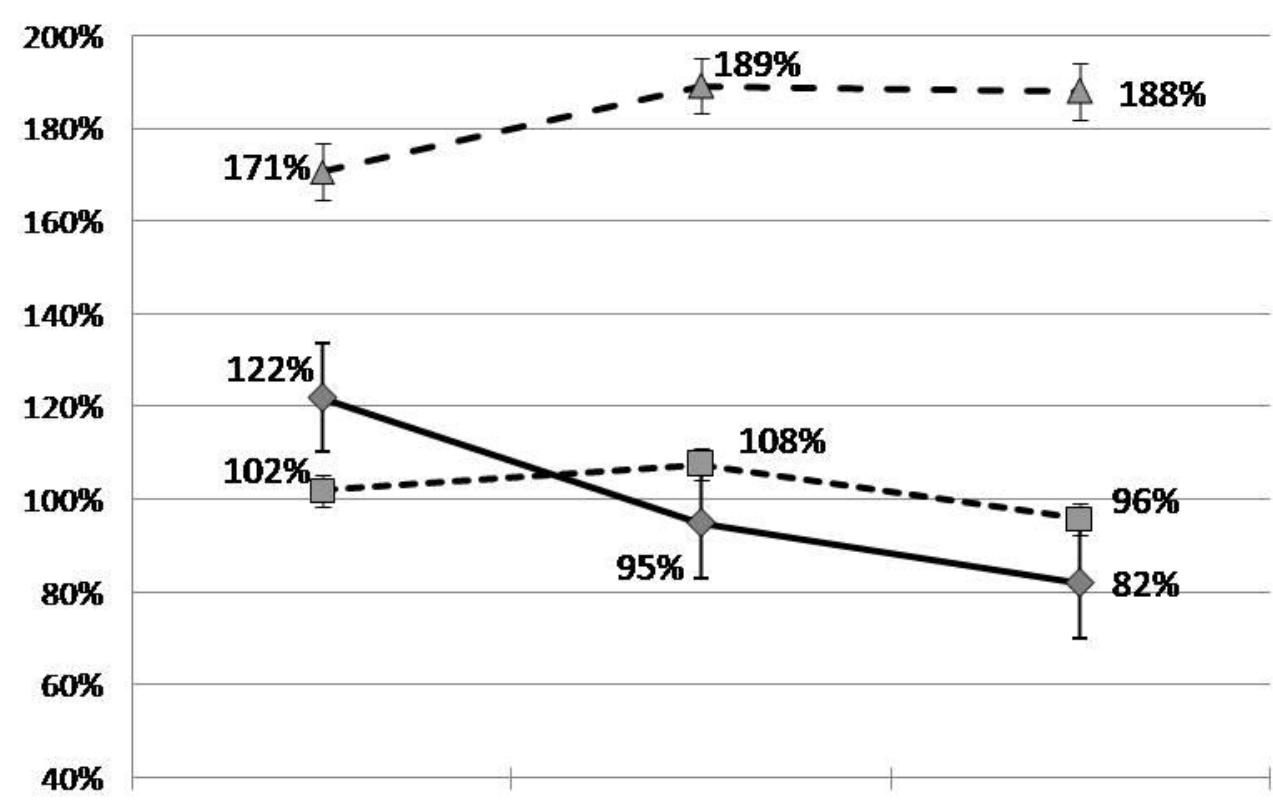

T1

12

T3

$\longrightarrow$ AMAN RCF $\quad-\square-$ AMANAX DEG $\quad-\triangle$ AIDP 